\title{
Behcet disease presenting initially as isolated recurrent aphthous ulcers only: A case report
}

\author{
Dickson Sopuru Okoh ${ }^{1 *}$, Pedro Emualosi ${ }^{1}$, Isaac Izevbigie ${ }^{1}$ and Mercy Okoh ${ }^{2}$ \\ ${ }^{1}$ Department of Dental and Maxillofacial Surgery, Federal Medical Centre, Asaba, Delta State, Nigeria \\ ${ }^{2}$ Department of Oral Pathology and Medicine, University of Benin, Benin City, Edo State, Nigeria
}

\begin{abstract}
Background: Behcet syndrome is a chronic multisystem vasculitis of unknown origin with frequent manifestation of oral and genital ulcerations, ocular and skin lesions and other involvement of the central nervous system and the gastrointestinal tract. We present a case of Behcet syndrome presenting initially with isolated oral aphthous ulcers and the aim is to highlight the clinical features and its treatment.

Case Report: A 36 year old female with recurrent aphthous ulcers on the tongue for 2 days with a history of frequent recurrence. Patient responded initially to topical triamcinolone acetonide and low dose prednisolone. Two weeks later, there was a recurrence of oral aphthous with erosions on the oral mucosa with skin and genitalial lesions. A diagnosis of Erythema Multiforme major was made and patient placed on higher dose steroid with resultant healing. There was another recurrence with lip crusting, sore throat, skin lesions, conjuctival inflammation and genitalia ulcerations. We made a diagnosis of Behcet's syndrome. Patient was placed on Dapsone resulting in complete resolution of the lesions with no recurrence within and after three months of several recall visits.
\end{abstract}

Conclusion: A case of Behcet's syndrome that affected the oral mucosa at initially and later the conjunctiva, the skin, and the genitalia in a 36 year-old female. Health professionals should be aware of this rare condition.

\section{Introduction}

Behçet's disease or behcets syndrome also known as Silk Road disease is a rare systemic vasculitis disorder of unknown etiology characterized by recurrent acute inflammatory attacks [1]. It is a chronic, relapsing, multisystem inflammatory disorder [2]. It was named after Hulusi Behcet, a Turkish dermatologist who in 1937 presented three cases of patients he followed for years with a triad of oral ulceration, genital lesions, and recurrent eye inflammation $[3,4]$. Zeidan, et al. [1] referred to this manifestations as the triple-symptom complex. It can affect the skin, blood vessels, gastrointestinal tract, central nervous system, heart, and other organs, as well as causing aphthous ulcers, genital ulcers, and eye inflammation [5]. Behçet's disease (BD) is also considered to represent an autoimmune reaction triggered by a yet to be identified infectious agent in a genetically predisposed person [6]. Multiple criteria have been created as guides for diagnosis; however, given the wide spectrum of organ involvement, some cases remain undiagnosed. The diagnosis of Behcet's Disease may only be made over time as the clinical manifestations unfold sometimes separated by months and even years [3]. Genetic studies have identified HLA-B ${ }^{\star} 51$ to be the important genetic risk factor [7]. There is a close correlation between the geographical distribution of HLA-B51 and its prevalence. In the aetiopathogenesis there are indications of genetic susceptibility associated with environmental influence [8].

Prevalence of BD worldwide ranges between $0.1 / 1000$ and 1/10,000 with a significant presence in Asian countries $30^{\circ}-40^{\circ}$ north of the Equator from the Mediterranean to Japan [1]. Cases of BD seem to cluster along the ancient Silk Road, which extends from eastern Asia to the Mediterranean basin. The prevalence is 80 to 370 cases per 100,000 population in Turkey having the highest reported prevalence, $10 / 100,000$ in Japan and $0.6 / 100,000$ in Yorkshire [4]. It remains rare in other countries such as the Americas including the Caribbeans and African countries [2,3]. Onset of Behcets mostly peaks in the third decade [8]. The male to female ratio is 7:1 in symptomatic forms, but women predominate over men in studies were less symptomatic forms are systematically looked for and included $[4,9]$. Though oral aphthous ulcers are a frequent finding in in patients with Behcet's, the diagnosis of this condition may be challenging when oral aphthous ulcers are the only persistent clinical manifestation at various times of presentation without the appearance or a history of the occurrence of the other component clinical features of this disease.

We present a case of Behcet disease in a patient who presented to us initially with frequent persistent isolated occurrence of oral aphthous ulcers only and to highlight the challenges associated with the diagnosis of Behcet's disease occurring initially as isolated recurrent aphthous ulcers and the importance of early diagnosis and treatment of the lesion.

\section{Case report}

A case of a 36 year old married female fashion designer who first presented 2 years ago to the Dental clinic, Department of Dental and Maxillofacial Surgery, Federal Medical Centre, Asaba, Delta State with

${ }^{\star}$ Correspondence to: Okoh DS, Department of Dental and Maxillofacial Surgery, Federal Medical Centre, Asaba, Delta State, Nigeria, Tel: +234805850035; E-mail: okodick@yahoo.com

Key words: aphthous ulcer, diagnosis, Behcet's syndrome, dapsone

Received: October 06, 2018; Accepted: October 17, 2018; Published: November 03,2018 
a one year history of frequent recurring oral ulcerations. The patient was managed on account of recurrent aphthous ulcers. She was placed on topical steroid-triamcinolone acetonide $0.1 \%$ dental paste three times daily for 2 days, Tablet augmentin (amoxicillin and clavulinic acid combination) $625 \mathrm{mg} 12$ hourly for 5 days, metronidazole $400 \mathrm{mg}$ 8 hourly for 5 days and paracetamol 1000mg three times daily for 3 days. Following several follow up visits, the ulcers healed. After one year, the patient again presented to the Dental clinic with recurrent aphthous ulcers on the tongue of 2 days duration and was referred to the clinical oral pathology and oral medicine clinic of the Department.

On examination, the ulcers were oval with erythematous margins and pale floor. The patient also complained of abdominal pain. She was placed on topical triamcinolone acetonide twice daily for 5 days, low dose systemic steroid tablet Prednisolone $10 \mathrm{mg}$ daily for 5 days followed by $5 \mathrm{mg}$ for the next 5 days, tetracycline mouth wash 4 times daily for 5 days, Xylonor gel, and tablets paracetamol 1000mg 3 times daily for 3 days. Patient responded well with healing of the ulcers after the steroid therapy. She was also seen by the gastroenterology unit where she was managed for dyspepsia. She was on omeprazole tablet $20 \mathrm{mg}$ twice daily for 2 weeks and suspension Gestid $10 \mathrm{mls} 3$ times daily for 1 week. However, just after 2 weeks, there was a recurrence of the oral aphthous ulcers with erosions on the ventral surface of the tongue and the right angle of the mouth, white patchy areas on the right buccal mucosa, target lesions on the skin and genitalial lesions (Figure 1).

Prior to this visit, she had been seen at the gynaecology clinic where she was managed on account of vulvovaginitis which was characterized by vaginal discharge, vulval itching and genital ulcer. We made a diagnosis of Erythema Multiforme major and patient was placed on a higher dose of systemic steroid, tablet Prednisolone 30mg daily for 5 days which was tapered down gradually by $5 \mathrm{mg}$ after every five days, acyclovir tablet $400 \mathrm{mg} 4$ times daily for 5 days and to continue the topical triamcinolone acetonide. There was resultant healing of the oral ulcers and erosions, skin lesions and the genital ulcers after two weeks of treatment. Shortly again, there was another recurrence after 3 weeks with manifestations of lip crusting, sore throat, skin lesions including papulopustular rashes with areas of depigmentation and hyperpigmentation, genitalia ulcerations, conjuctival inflammation with redness, eye pain and poor vision, and associated abdominal pain (Figure 2). Based on these findings and the criteria for diagnosis, we made a diagnosis of Behcet's syndrome. Due to the prolong use of the systemic steroid, patient was alternatively placed on Dapsone $25 \mathrm{mg}$ twice daily for 1 week and to continue with the topical triamcinolone acetonide. Patient was also referred to the ophthalmology clinic where she was managed for conjuctival inflammation and uveitis. After the duration of the Dapsone therapy, there was complete resolution of the oral, skin, genitalial and eye lesions with no recurrence within three months of several recall visits, and patient has not reported with any complaints long after the recall visits (Figure 3).

\section{Discussion}

The clinical characteristics and symptoms are the main factors for diagnosing Behcet Disease, but a specific diagnostic pathognomonic feature or laboratory method is not yet available [10]. Diagnosis of clinical symptoms is challenging, especially when symptoms are nonconcomitant [1]. Diagnosis of Bechet Syndrome is based on clinical criteria and hence several criteria have been proposed such as that by the international study for Behcet (ISG) in 1990 and the International Criteria for Behcet's Disease (ICBD) [11,12]. In 2012, the international Chapel Hill's 1990 criteria were revised and a new proposal was made. The diagnosis is made by the presence of recurrent oral ulceration (at

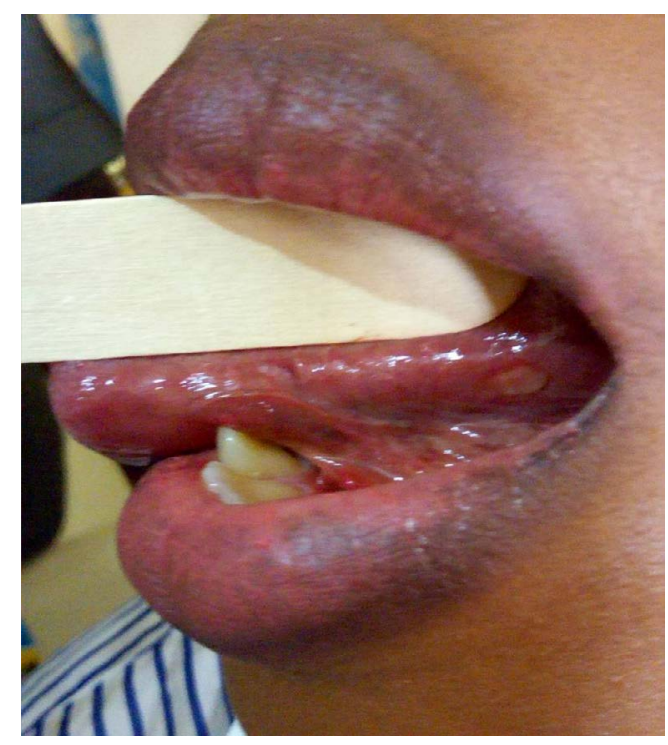

Figure 1. Major Apthous ulcer on the lateral border of the tongue

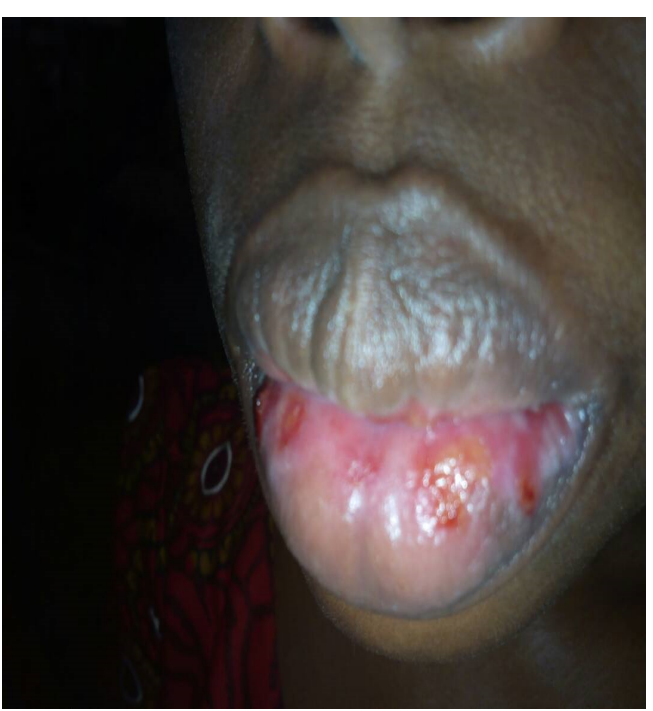

Figure 2. Multiple erythemas, ulcers and erosions with crusting on the lower lip

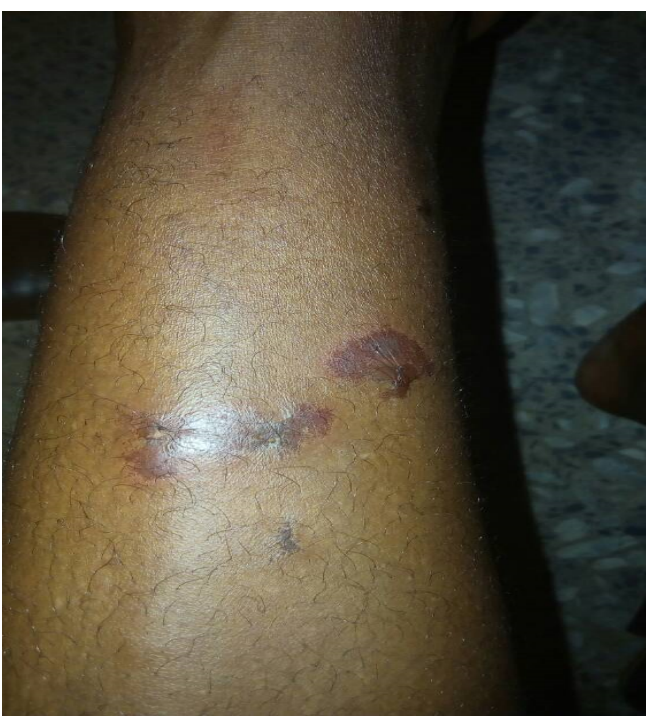

Figure 3. Hyperpigmented and erythema nodosum-like skin lesions on the lower extremities 
least three times a year) plus any two of the following: recurrent genital ulceration (leaving scars), ocular lesions (anterior uveitis, posterior uveitis, presence of cells in the vitreous detected in the slit lamp examination, retinal vasculitis), cutaneous lesions (erythema nodosum, pseudofolliculitis or papulopustular lesions, acneiform nodules in patients after adolescence and who are not using corticosteroids), or positive pathergy test results with reading performed 24-48 hours after the test $[1,8]$ (Figure 4 ). The prevalence of Bechets disease in Nigeria and other parts of sub-Saharan Africa is not fully known owing to the paucity of reported cases of Behcet. However, Poon, et al. [2] stated that that the prevalence of Behçet's disease is low both in central and West African populations. Although BD affects both genders worldwide and in sub-Saharan Africa, most studies have however reported more occurrences in males [6,13-15]. Our patient was a female patient who presented in her fourth decade which is within the age range of between 18 to 40 years stated by David Saadoun [4] but contrast with reports that showed peak incidence in the third decade $[8,16]$. Since our report is on a case and due to the rarity of this condition, we cannot conclude on the age and gender pattern in our environment. A case series involving a large number of patients would help to determine the pattern in our environment. This patient had a multidisciplinary treatment involving the oral pathology and oral medicine unit, gastroenterologist and the gynaecologist. Management of Bechets syndrome is a multidisciplinary approach to reduce morbidity and mortality [16]. Behcets Disease responds to corticosteroids and immunosuppressive therapy $[1,15,16]$. The beneficial effects of Dapsone have been reported [17]. Sharquie, et al. [18] investigated the effect of Dapsone in the treatment of mucocutaneous manifestations of Behcet's disease and the possible prophylactic role of Dapsone in a double blind/placebo controlled clinical trial involving 20 patients. Those that had Dapsone had a significant reduction in the frequency and duration of oral and genital ulcers than those on placebo. Dapsone inhibits the enhanced chemotactic activity of neutrophils [19]. Hemolytic anemia and methemoglobinemia, which can be severe in patients with glucose6-phosphate dehydrogenase deficiency, are the main side effects, which

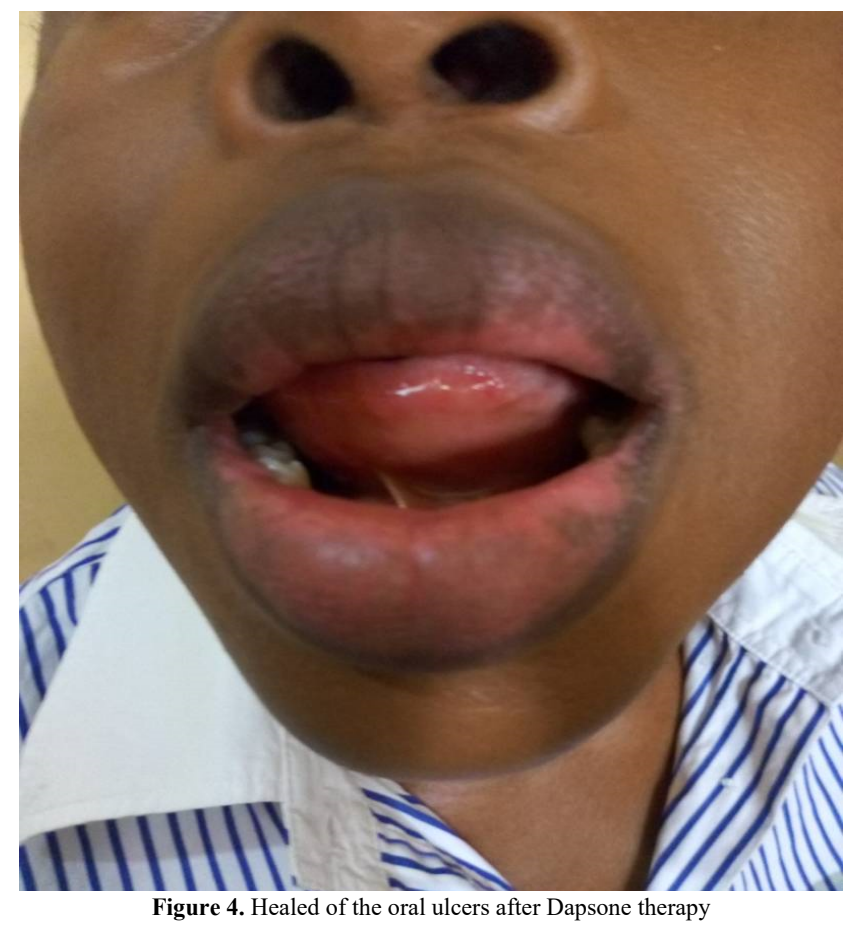

may significantly limit their use [19]. The patient in the present study presented with no adverse reactions to the use of Dapsone.

Some differential diagnosis of Bechets at different stages of manifestations includes recurrent aphthous stomatitis, recurrent herpetic stomatitis, erythema multiforme, Steven Johnson syndrome and complex aphthosis. Recurrent Aphthous Stomatitis occurs in patients who are otherwise well, affecting the oral mucosa and characterized by oval ulcers with erythematous margins [20]. In Recurrent herpetic stomatitis, patient would have given a history of fluid filled vesicle formation that ruptured. The individual lesions begin as vesicles on mucocutaneous areas which quickly burst, leaving punctate ulceration with an erythematous base [21]. The most common type of recurrent herpetic stomatitis are presence of small blisters on the lips commonly referred to as fever blisters or secondary herpes stomatitis which affects mostly the lips [22]. Hypersensitivity reactions like Erythema multiforme show crusting and haemorrhagic ulceration with multiple erythematous patches on the lip and oral mucosa with target lesions on the skin [23]. A more severe spectrum of erythema multiforme, the Steven Johnson's syndrome is a hypersensitivity reaction and affecting mucocutaneous surfaces characterized by nonspecific febrile illness (malaise, headache, cough, rhinorrhea) with polymorphic lesions of skin and mucous membrane characterized by acute blisters and erosions [24]. Behcets is a much more severe disease than complex aphthosis. Complex aphthosis is an uncommon, persistent, chronic type of recurrent aphthous stomatitis which may also be associated with systemic diseases and patients may also have anogenital aphthae [25].

\section{Conclusion}

We report a case of Behcet's syndrome, a rare clinical condition that affected the oral mucosa, the conjunctiva, the skin, the genitalia and gastrointestinal tract in a 36 year-old female that presented to us initially with just recurrent aphthous ulcers only. There was positive response after the use of corticosteroid initially and Dapsone treatment. Healthcare professionals should be aware of this rare condition for prompt diagnosis and adequate treatment.

\section{Reference}

1. Zeidan MJ, Saadoun D, Garrido M, Klatzmann D, Six A, et al. (2016) Behçet's disease physiopathology: a contemporary review. Auto Immun Highlights 7: 4.

2. Poon W, Verity DH, Larkin GL, Graham EM, Stanford MR (2003) Behçet's disease in patients of west African and Afro-Caribbean origin. Br J Ophthalmol 87: 876-878. [Crossref]

3. Leonardo NM, McNeil J (2015) Behçet's disease: is there geographical variation? A review far from the Silk Road. Int J Rheumatol.

4. Saadoun D, Wechsler B (2012) "Behçet's disease”. Orphanet J Rare Dis 7: 20

5. Jun JH, Choi TY, Zhang J, Ko MM, Lee MS (2018) Herbal medicine for Behcet's disease: A protocol for a systematic review and meta-analysis. Medicine (Baltimore) 97: 13

6. Ajose F, Adelowo, Oderinlo (2015) Clinical presentations of Behçet's disease among Nigerians: a 4-year prospective study. Int J Dermatol 54: 889-897.

7. HasanYazici, EmireSeyahi, GulenHatemi, Yusuf Yazici (2018) Behçet syndrome: a contemporary view. Nat Rev Rheumatol 14: 107-119.

8. Scherrer MAR, Rocha VB, Garcia LC (2017) Behçet's disease: review with emphasis on dermatological aspects. An Bras Dermatol 92: 452-464. [Crossref]

9. Saadoun D, Wechsler B, Desseaux K, Le Thi Huong D, Amoura Z, et al. (2010) Mortality in Behçet's disease. Arthritis Rheum 62: 2806-2812. [Crossref]

10. Ertaş R, Özyurt K, Avcı A, Ertas SK, Atasoy M (2017) Case Report: Behçet's disease accompanied with vitiligo. F1000Res $6: 310$. [Crossref] 
11. International Study Group for Behçet's Disease (1990) Criteria for diagnosis of Behçet's disease. Lancet 335: 1078-1080. [Crossref]

12. International Team for the Revision of the International Criteria for Behçet's Disease (ITR-ICBD) (2014) The International Criteria for Behçet's Disease (ICBD): a collaborative study of 27 countries on the sensitivity and specificity of the new criteria. J Eur Acad Dermatol Venereol 28: 338-347. [Crossref]

13. Dawodu CO, Adeleye OA (2010) Neurological Manifestation of Behcet's Disease: A Case Report. Niger J Clin Med 2: 2

14. Meda JR, Seni J, Mpondo B, Peck RN, Jaka H, et al. (2014) Behcet's disease presenting with recurrent ocular, oral, and scrotal inflammatory lesions in a young Tanzanian man: a case report. Clin Case Rep 2: 133-136.

15. Adeoti AO, Ibidapo OR, Ojo O (2017) Pulmonary Artery Aneurysm in Behcet's Disease: A Ticking Time Bomb. Clin Med Case Re 7: 201.

16. Hatemi G, Seyahi E, Fresko I, Talarico R, Hamuryudan V (2015) Behçet's syndrome: a critical digest of the 2014-2015 literature. Clin Exp Rheumatol 33: S3-14. [Crossref]

17. Sharquie KE (1984) Suppression of Behçet's disease with dapsone. Br J Dermatol 110 493-494. [Crossref]
18. Sharquie KE, Najim RA, Abu-Raghif AR (2002) Dapsone in Behçet's disease: a double-blind, placebo-controlled, cross-over study. J Dermatol 29: 267-279. [Crossref]

19. Erkan Alpsoy (2012) "New Evidence-Based Treatment Approach in Behçet’s Disease," Patholog Res Int p. 11.

20. Preeti L, Magesh K, Rajkumar K, Karthik R (2011) Recurrent aphthous stomatitis. $J$ Oral Maxillofac Pathol 15: 252-256.

21. Han-Seok Kim, Suk-Keun Lee, Young-Wook Park (2011) Recurrent Herpes-Stomatitis Mimicking Acute Necrotizing Ulcerative Gingivitis. J Korean Assoc Maxillofac Plast Reconstr Surg 33: 89-92.

22. Sumit Bhateja, Geetika Arora (2017) Recurrent Intraoral Herpes (RIH) Infection - A Case Report. Biomed J Sci \& Tech Res p. 1.

23. Omoregie FO, Ukpebor M, Saheeb BD (2011) Methotrexate-induced erythema multiforme: a case report and review of the literature. West Afr J Med 30: 377-379. [Crossref]

24. Balasundaram S, Ranganathan K, Umadevi K (2011) Oral lesions associated with nevirapine-related Stevens Johnson syndrome: A report of four cases. J Oral Maxillofac Pathol 15: 39-45.

25. Rogers RS (2004) Complex Aphthosis. In: Zouboulis CC (eds) Adamantiades-Behçet's Disease. Adv Exp Med Biol. Springer, Boston, MA.

Copyright: (C2018 Okoh DS. This is an open-access article distributed under the terms of the Creative Commons Attribution License, which permits unrestricted use, distribution, and reproduction in any medium, provided the original author and source are credited. 